\title{
Pinocchio: Nearly Practical Verifiable Computation
}

Bryan Pano, Jon Howell, Craig Gentry, Mariana Raykova

Presentated by Phd@ECE,UMD
Hui Zhang

wayne.huizhang@gmail.com 


\section{Introduction}

- Outsourcing complex computation to powerful servers is becoming popular.

- However, the workers that help the client to do the job is not always reliable: malicious or malfunctioning workers.

- Previous work has many shortages: impractical time consumption, function specific, not public verification, not zero-knowledge, etc. 


\section{Related works}

- Function specific solutions [2-6] are often efficient, but only for a narrow class of computations. More general solutions often rely on assumptions that may not apply. For example, systems based on replication $[1,7,8]$ assume uncorrelated failures, while those based on Trusted Computing [9-11] or other secure hardware [12-15] assume that physical protections cannot be defeated. Finally, the theory community has produced a number of beautiful, general purpose protocols [16-23] that offer compelling asymptotics. In practice however, because they rely on complex Probabilistically Checkable Proofs (PCPs) [17] or fullyhomomorphic encryption (FHE) [24], the performance is unacceptable -verifying small instances would take hundreds to trillions of years. Very recent work [25-28] has improved these protocols considerably, but efficiency is still problematic, and the protocols lack features like public verification. 


\section{Contributions of the paper}

1. An end-to-end system for efficiently verifying computation performed by one or more untrusted workers. This includes a compiler that converts "C" code into a format suitable for verification, as well as a suite of tools for running the actual protocol.

- We'll see how to use the tool later 


\section{Contributions}

2. Theoretical and systems-level improvements that bring time down by 5-7 orders of magnitude, and hence into the realm of plausibility. The proof is only $\mathbf{2 8 8}$ bytes, regardless of the computation performed or the size of the inputs and outputs.

3. An evaluation on seven real $\mathrm{C}$ applications, showing verification faster than 32 -bit native integer execution for some apps. 


\section{Background Knowledge}

- Verifiable Computation (VC)

A public verifiable computation (VC) scheme allows a computationally limited client to outsource to a worker the evaluation of a function $F$ on input $u$. The client can then verify the correctness of the returned result $F(u)$ while performing less work than required for native execution. 


\section{Public Verifiable Computation}

- $\left(E K_{F}, V K_{F}\right) \leftarrow \operatorname{KeyGen}\left(F, 1^{\lambda}\right)$ :

- $\left(y, \pi_{y}\right) \leftarrow$ Compute $\left(E K_{F}, u\right)$ :

- $\{0,1\} \leftarrow \operatorname{Verify}\left(V K_{F}, u, y, \pi_{y}\right)$ : 


\section{Correctness, Security, Efficiency}

- Correctness For any function $F$, and any input $u$ to $F$, if we run $\left(E K_{F}, V K_{F}\right) \leftarrow \operatorname{KeyGen}\left(F, 1^{\lambda}\right)$ and $\left(y, \pi_{y}\right) \leftarrow$ Compute $\left(E K_{F}, u\right)$, then we always get $1=$ $\operatorname{Verify}\left(V K_{F}, u, y, \pi_{y}\right)$.

- Security For any function $F$ and any probabilistic polynomial-time adversary $\mathcal{A}$, $\operatorname{Pr}\left[\left(\hat{u}, \hat{y}, \hat{\pi}_{y}\right) \leftarrow \mathcal{A}\left(E K_{F}, V K_{F}\right): F(\hat{u}) \neq \hat{y} \quad\right.$ and $\left.1=\operatorname{Verify}\left(V K_{F}, \hat{u}, \hat{y}, \hat{\pi}_{y}\right)\right] \leq \operatorname{negl}(\lambda)$.

- Efficiency KeyGen is assumed to be a one-time operation whose cost is amortized over many calculations, but we require that Verify is cheaper than evaluating $F$. 


\section{Zero-knowledge Verifiable Computation}

- $\mathrm{F}(\mathrm{u} ; \mathrm{w})$, of two inputs: the client's input $u$ and an auxiliary input $w$ from the worker.

- A VC scheme is zero-knowledge if the client learns nothing about the worker's input beyond the output of the computation 


\section{Quadratic Programs}

- GGPR[2] has shown how to compactly encode computations as quadratic programs, so as to obtain efficient VC and zero-knowledge VC scheme.

- Specifically, they show how to convert any arithmetic circuit into a comparably sized QAP, and any Boolean circuit into a comparably sized QSP 


\section{Arithmetic Circuits and QAPs}

An arithmetic circuit consists of wires that carry values from

a field $\mathrm{F}$ and connect to addition and multiplication gates

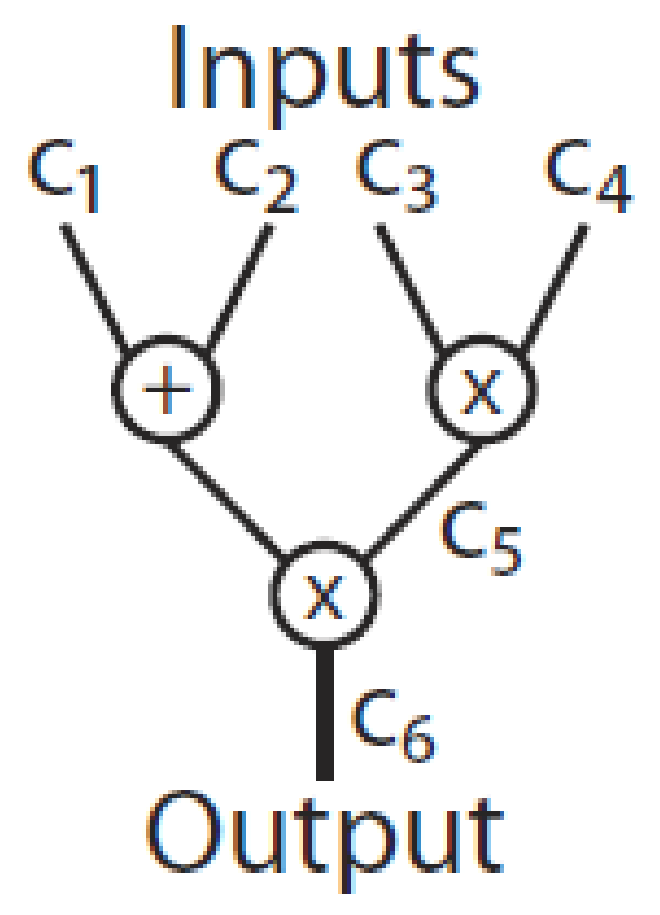




\section{Definition (QAP)}

- QAP: an encoding of an Arithmetic Circuit

A QAP $Q$ over field $\mathbb{F}$ contains three sets of $m+1$ polynomials $\mathcal{V}=\left\{v_{k}(x)\right\}, \mathcal{W}=\left\{w_{k}(x)\right\}, \mathcal{Y}=\left\{y_{k}(x)\right\}$, for $k \in\{0 \ldots m\}$, and a target polynomial $t(x)$. Suppose $F$ is a function that takes as input $n$ elements of $\mathbb{F}$ and outputs $n^{\prime}$ elements, for a total of $N=n+n^{\prime} I / O$ elements. Then we say that $Q$ computes $F$ if: $\left(c_{1}, \ldots, c_{N}\right) \in \mathbb{F}^{N}$ is a valid assignment of $F$ 's inputs and outputs, if and only if there exist coefficients $\left(c_{N+1}, \ldots, c_{m}\right)$ such that $t(x)$ divides $p(x)$, where:

$$
\begin{aligned}
p(x)= & \left(v_{0}(x)+\sum_{k=1}^{m} c_{k} \cdot v_{k}(x)\right) \cdot\left(w_{0}(x)+\sum_{k=1}^{m} c_{k} \cdot w_{k}(x)\right) \\
& -\left(y_{0}(x)+\sum_{k=1}^{m} c_{k} \cdot y_{k}(x)\right) .
\end{aligned}
$$




\section{Building a QAP}

1. Pick an arbitrary root $r_{g} \in F$ for each multiplication gate $\mathrm{g}$ in $\mathrm{C}$ and define the target polynomial to be $t(x)=\prod g\left(x-r_{g}\right)$

2. Associate an index $\mathrm{k}=\{1,2 \ldots, \mathrm{m}\}$ to each input of the circuit and to each output from a multiplication gate

3. Define three sets V, W, Y: V encode left input to each gate, $\mathrm{W}$ encode right input, $\mathrm{Y}$ encode the output 


\section{Building a QAP}

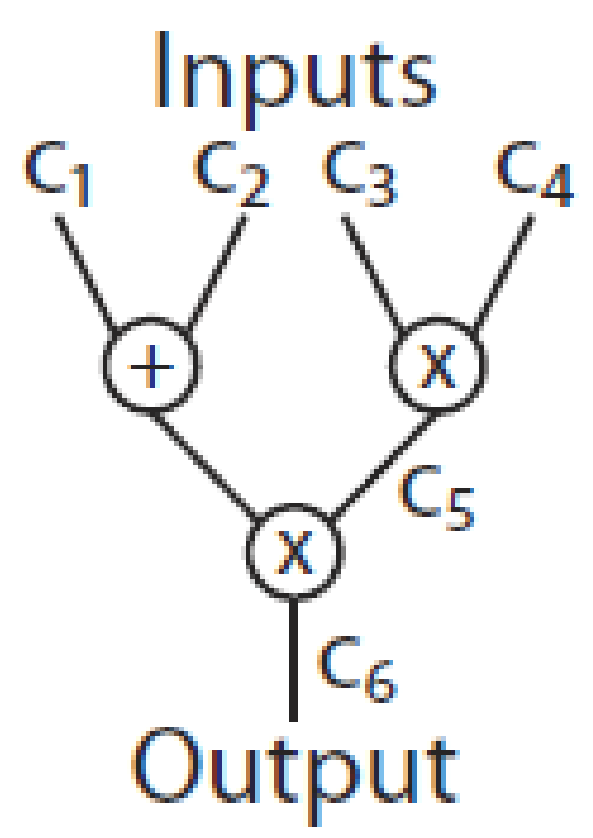

\begin{tabular}{lc|lc|lc} 
& $\left(r_{5}, r_{6}\right)$ & & $\left(r_{5}, r_{6}\right)$ & & $\left(r_{5}, r_{6}\right)$ \\
\hline$v_{1}\left(r_{i}\right)$ & $(0,1)$ & $w_{1}\left(r_{i}\right)$ & $(0,0)$ & $y_{1}\left(r_{i}\right)$ & $(0,0)$ \\
$v_{2}\left(r_{i}\right)$ & $(0,1)$ & $w_{2}\left(r_{i}\right)$ & $(0,0)$ & $y_{2}\left(r_{i}\right)$ & $(0,0)$ \\
$v_{3}\left(r_{i}\right)$ & $(1,0)$ & $w_{3}\left(r_{i}\right)$ & $(0,0)$ & $y_{3}\left(r_{i}\right)$ & $(0,0)$ \\
$v_{4}\left(r_{i}\right)$ & $(0,0)$ & $w_{4}\left(r_{i}\right)$ & $(1,0)$ & $y_{4}\left(r_{i}\right)$ & $(0,0)$ \\
$v_{5}\left(r_{i}\right)$ & $(0,0)$ & $w_{5}\left(r_{i}\right)$ & $(0,1)$ & $y_{5}\left(r_{i}\right)$ & $(1,0)$ \\
$v_{6}\left(r_{i}\right)$ & $(0,0)$ & $w_{6}\left(r_{i}\right)$ & $(0,0)$ & $y_{6}\left(r_{i}\right)$ & $(0,1)$
\end{tabular}

$$
t(x)=\left(x-r_{5}\right)\left(x-r_{6}\right)
$$




\section{Boolean Circuits and QSPS}

- Boolean circuits operate over bits, with bitwise gates for AND, OR, XOR, etc. GGPR propose Quadratic Span Programs (QSPs) as a custom encoding for Boolean circuits

- QSPs are superficially similar to QAPs, but because they only support Boolean wire values, they use only two sets of polynomials $\mathrm{V}$ and $\mathrm{W}$. 


\section{Theoretical Refinements}

- Originally, we build VC from strong QAPs

- Its main optimization is that we construct a $\mathrm{VC}$ scheme that uses a regular QAP, rather than a strong QAP.

- They also remove the need for the worker to compute gah(s), and hence the gasii2[d] terms from EK. Finally, we expand the expressivity and efficiency of the functions QAPs can compute by designing a number of custom circuit gates for specialized functions. 


\section{Performance}

- Proof size: 288-byte, regardless of the size of the computation

- Proof verification: 5 7 orders of magnitude performance improvement over prior work.

- Proof generation: 19-60 x faster.

- Cutting the cost of both key and proof generation by more than $60 \%$. 


\section{Performances}

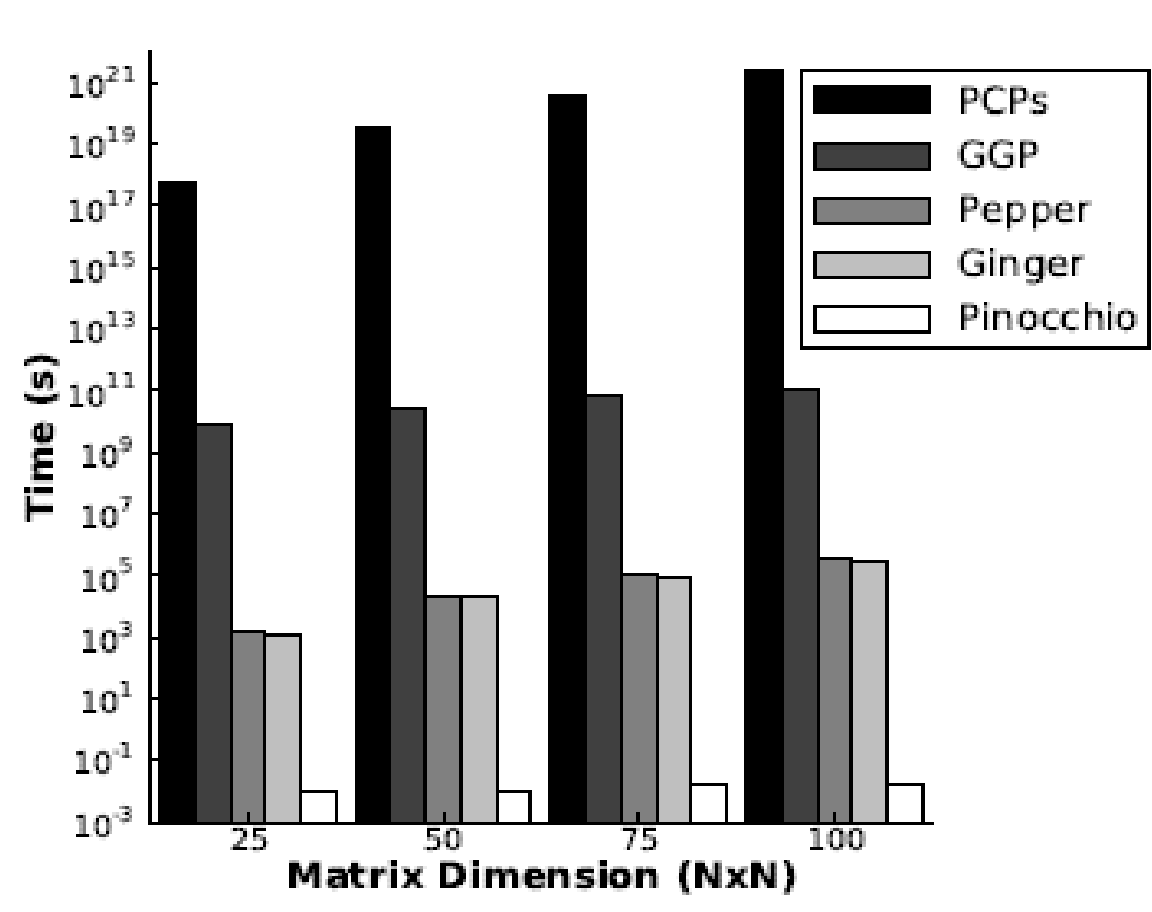

(a) Per-Instance Verification Latency

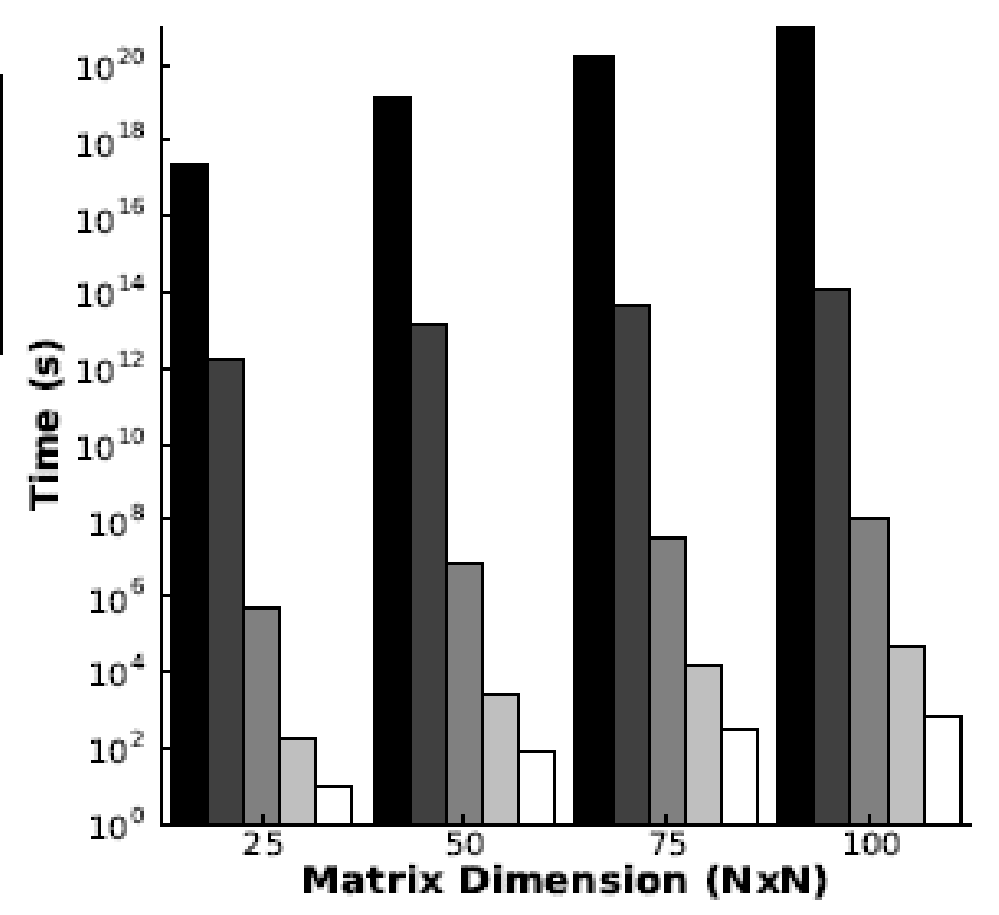

(b) Worker Latency

Figure 6: Performance Relative to Prior Schemes. Pinocchio reduces costs by orders of magnitude (note the log scale on the $y$ axis). We graph the time necessary to (a) verify and (b) produce a proof result for multiplying two $N x N$ matrices. 


\section{Performances}

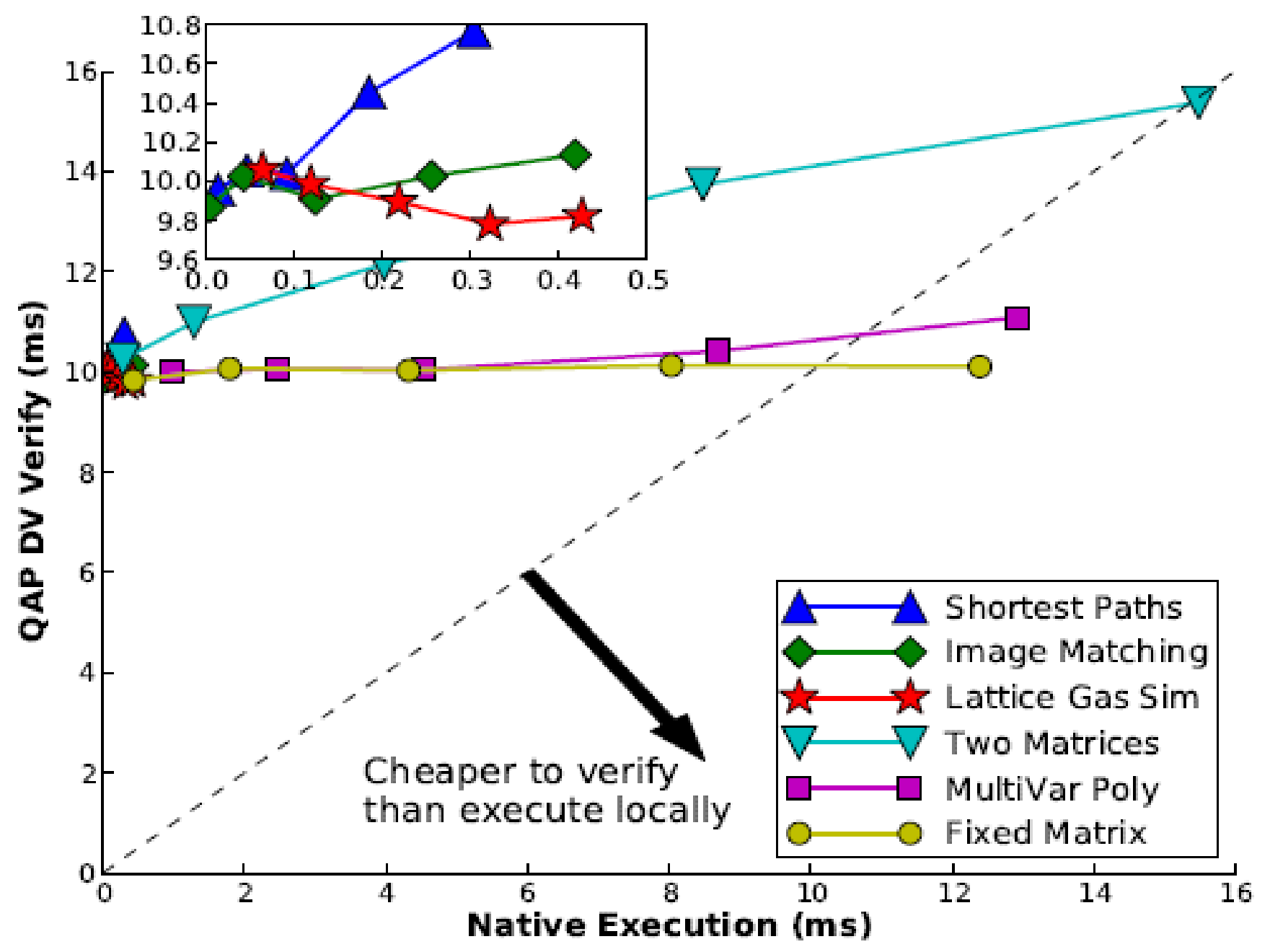




\section{Tool Usage}

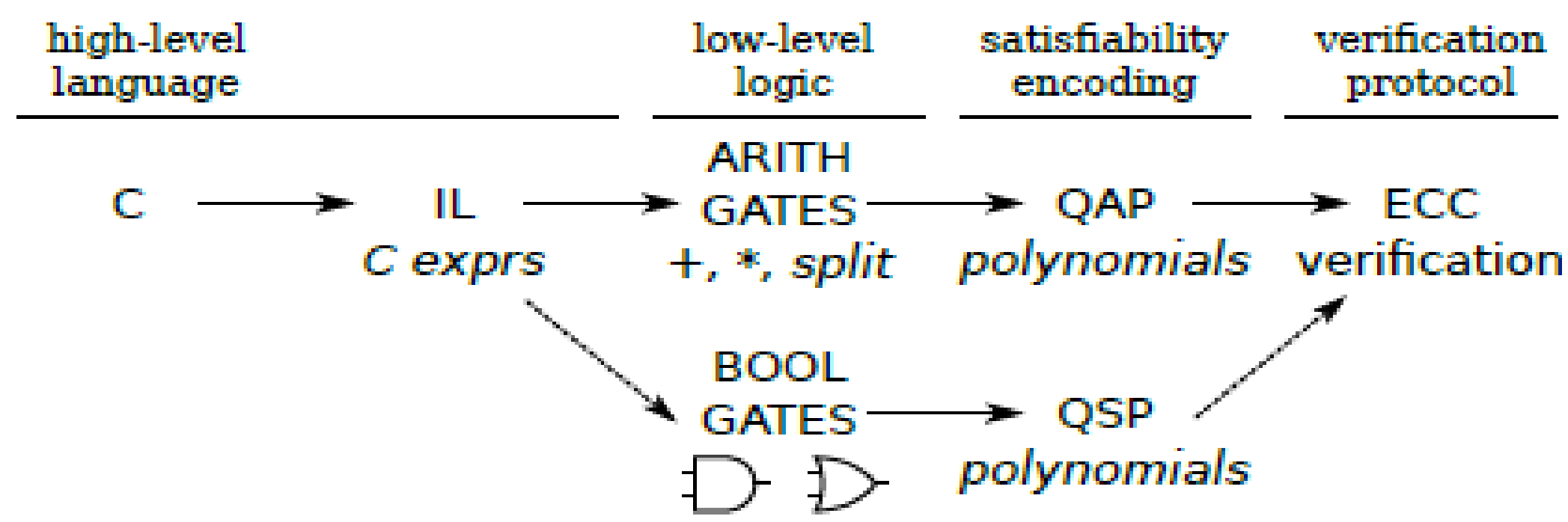

Figure 1: Overview of Pinocchio's Toolchain. Pinocchio takes a high-level C program all the way through to a distributed set of executables that run the program in a verified fashion. It supports both arithmetic circuits, via Quadratic Arithmetic Programs (\$2.2.1), and Boolean circuits via Quadratic Span Programs (§2.2.2). 


\section{Steps:}

- First step: Generate a .arith file that describes the circuit you will be operating on.

- 1) go to common/, change corresponding parameters and name of file you wan to test in App.py

- 2) go to ccompiler/input, run ../src/build-testmatrix.py

-3) run make -f make.matrix

- Second step: Run Pinocchio using the arith file obtained from the previous step

- pinocchio-vo.4.exe --qap --pv --bits 32 --mem 1 --file /path/to/.arith 
Q

\section{Thank you !}




\section{Reference}

- [1] Parno, Bryan, et al. "Pinocchio: Nearly practical verifiable computation."Security and Privacy (SP), 2013 IEEE Symposium on. IEEE, 2013.

- [2] Gennaro, Rosario, et al. "Quadratic span programs and succinct NIZKs without PCPs." Advances in Cryptology-EUROCRYPT 2013. Springer Berlin Heidelberg, 2013. 626-645. 